MISS MARIANNA LOVÁSZI (Orcid ID : 0000-0002-1353-5317)

DR STEFANIE EYERICH (Orcid ID : 0000-0002-1166-2355)

Article type : Original Article

\title{
Sebum lipids influence macrophage polarization and activation
}

Running head: Sebocyte produced lipids regulate macrophages

Manuscript word count: 3533 (materials and methods not included) table count: - and figure count: 4

M. Lovászi ${ }^{1}$, M. Mattii ${ }^{2}$, K. Eyerich ${ }^{3}$, Attila Gácsi ${ }^{4}$, Erzsébet Csányi ${ }^{4}$, D. Kovács ${ }^{1}$, R. Rühl ${ }^{5}$, A. Szegedi $^{1,6}$, L. Kemény ${ }^{7,8}$, M. Ståhle ${ }^{9}$, C. C. Zouboulis ${ }^{10,+}$, S. Eyerich $^{2}$, D. Töröcsik ${ }^{1,9+}$

${ }^{1}$ Department of Dermatology, Faculty of Medicine, University of Debrecen, Debrecen, Hungary

${ }^{2}$ ZAUM - Centre for Allergy and Environment, Technische Universität and Helmholtz Centre Munich, Member of the German Centre for Lung Research (DZL), Munich, Germany

${ }^{3}$ Department of Dermatology and Allergy, Technische Universität Munich, Munich, Germany

${ }^{4}$ Institute of Pharmaceutical Technology and Regulatory Affairs, Faculty of Pharmacy, University of Szeged, Szeged, Hungary

${ }^{5}$ MTA-DE Public Health Research Group of the Hungarian Academy of Sciences, Faculty of Public Health, University of Debrecen, Hungary

This article has been accepted for publication and undergone full peer review but has not been through the copyediting, typesetting, pagination and proofreading process, which may lead to differences between this version and the Version of Record. Please cite this article as doi: 10.1111/bjd.15754

This article is protected by copyright. All rights reserved. 
${ }^{6}$ Division of Dermatological Allergology, Faculty of Medicine, University of Debrecen, Debrecen, Hungary

${ }^{7}$ Department of Dermatology and Allergology, University of Szeged, Szeged, Hungary

${ }^{8}$ MTA-SZTE Dermatological Research Group, University of Szeged, Szeged, Hungary

${ }^{9}$ Unit of Dermatology and Venereology, Department of Medicine, Karolinska lnstitutet, Karolinska University Hospital, Stockholm, Sweden

${ }^{10}$ Departments of Dermatology, Venereology, Allergology and Immunology, Dessau Medical Centre, Dessau, Germany

${ }^{+}$D.T. and C.C.Z. contributed equally in the development of the concept of this work.

Correspondence: Dániel Törőcsik MD PhD, Department of Dermatology, Faculty of Medicine, University of Debrecen, Debrecen, Hungary, H4032, Nagyerdei krt. 98.

Telephone: +3652255602

Fax: +36 52255736

Email: dtorocsik@gmail.com

This work was supported by the Hungarian National Research Fund (OTKA NN117020), Marie Skłodowska-Curie actions (627547 RNAsebgland FP7-PEOPLE-2013-IEF), the New National Excellence Program of the Ministry of Human Capacities (D.T) and the Fondation Acteria and the Helmholtz Association (S.E). The funders had no role in study design, data collection and analysis, decision to publish, or preparation of the manuscript.

The authors have nothing to disclose.

This article is protected by copyright. All rights reserved. 
Bulleted statements

What's already known about this topic?

The primary function of human sebaceous glands is to produce and secrete sebum, which so far, was considered to only contribute to the lipid barrier of the skin.

What does this study add?

Our work indicates that sebocyte-derived lipids may also target macrophage differentiation and activation. Moreover, in the pathogenesis of acne, the Propionibacterium acnes - macrophage interaction might be largely dependent on the composition of the sebum, which is of possible pathologic and therapeutic relevance.

\section{Summary}

BACKGROUND: As lipids are known to regulate macrophage functions it is reasonable to suppose that a sebocyte - macrophage axis mediated by sebum lipids may exist.

OBJECTIVE: To investigate if sebocytes could contribute to the differentiation, polarization and function of macrophages with their secreted lipids.

METHODS: Oil-red-O lipid staining and Raman spectroscopy were used to assess the dermal lipid content and penetration. Immunohistochemistry was used to analyse the macrophage subsets. Human peripheral blood monocytes were differentiated in the presence of either supernatant from human SZ95 sebocytes or major sebum lipid components and activated with Propionibacterium acnes. Macrophage surface markers and their capacity to uptake FITC-Propionibacterium acnes were

This article is protected by copyright. All rights reserved. 
detected by FACS measurements. Cytokine protein levels were evaluated by ELISA and Western blot analysis.

RESULTS: Sebaceous gland rich skin had an increased dermal lipid content compared to sebaceous gland poor skin to which all the tested sebum component lipids could contribute by penetrating through the dermo-epidermal barrier. Of the lipids, oleic and linoleic acids promoted monocyte differentiation into alternatively activated macrophages. Moreover, linoleic acid also had an antiinflammatory effect in Propionibacterium acnes activated macrophages, inhibiting the secretion of IL-1B, IL-6 and TNF-A. Squalene, palmitic, stearic and oleic acids augmented the secretion of IL-1B even in the absence of Propionibacterium acnes, while oleic acid had a selective effect of inducing IL-1B, but down-regulating IL-6 and TNF-A secretion.

CONCLUSIONS: Our results suggest a role for sebaceous glands in modulating innate immune responses via their secreted lipids that are of possible pathologic and therapeutic relevance.

Key words: sebocyte, macrophage, sebum, Propionibacterium acnes

\section{Introduction}

The primary function of human sebaceous glands is considered to be the production and secretion of sebum to cover the hair and contribute to the lipid barrier of the skin ${ }^{1-3}$. Recent findings, however, have shown that sebocytes can also produce cytokines and inflammatory mediators both of protein and of lipid nature ${ }^{4}$ and are capable to alter their sebum production/composition in response to different inflammatory stimuli ${ }^{5-8}$. These findings already suggest that sebocytes may not just be a target of inflammation, as previously considered, but could actively contribute to skin homeostasis and the inflammatory environment $\mathrm{t}^{9-13}$.

This article is protected by copyright. All rights reserved. 
Macrophages, the key phagocytes of the immune system, sense and transform signals and stimuli towards the initiation of inflammation and activation of the adaptive immune system. In order to fulfil these complex roles, macrophages exert a prominent morphological and functional heterogeneity in both the healthy and the inflamed skin, outlining the importance of the different stimuli behind their activation $^{14}$. Based on these stimuli, a widely accepted classification separates macrophages into alternatively and classically activated subsets ${ }^{15}$. While alternative macrophages are activated by IL4/IL-13 and by various lipids that are associated with tissue maintenance and remodelling processes, and are increased in high numbers in pathological conditions such as wound healing or tumour matrix formation the classically activated macrophages typically develop in response to IFN-G and/or TNF$\mathrm{A}^{16,17}$.

In this study, we ex vivo assessed the extent in which sebocyte derived lipids may contribute to the dermal lipid content. While in an in vitro setting where human blood-derived monocytes/macrophages were cultured in the supernatant of human SZ95 sebocytes or selected sebum component lipids, we addressed the contribution of sebocyte derived lipids to macrophage polarization as well as to their interaction with Propionibacterium acnes (P. acnes) that is believed to be a pivotal step in the development of acne, the most common disease of the sebaceous glands ${ }^{18-20}$.

\section{Materials and methods}

\section{Oil-Red-O staining}

Anonymised frozen skin biopsies were sectioned and fixed in 4\% Paraformaldehyde (Sigma-Aldrich, Dorset, UK) for $10 \mathrm{~min}$. The sections were stained with 0,7\% Oil Red O (Sigma-Aldrich) solution for 7 minutes. A counter staining for nuclei was done with methylene green. Detailed staining protocol is available in supplementary information online.

This article is protected by copyright. All rights reserved. 


\section{Raman spectroscopy}

Skin biopsies were obtained from abdominal reconstruction surgery. Approximately $1 \mathrm{~cm}^{2}$ skin samples were treated with either squalene, linoleic-, oleic-, palmitic- or stearic acid for $24 \mathrm{~h}$, the treated area was $66.5 \mathrm{~mm}^{2}$ in all cases. Tissue samples of the treated areas were frozen and sectioned onto aluminium coated slides. Raman spectra of each section were obtained with a DXR Raman microscope (Thermo Fisher Scientific, Waltham, MA, USA) equipped with a 532nm diode laser. Instrument operation and the evaluation of measurements were done by the OMNIC Dispersive Raman 8.2 software (Thermo Fisher Scientific). The individual spectra of each fatty acid were used as a reference when comparing the treated vs untreated samples. Detailed protocol is available in supplementary information online.

\section{SZ95 sebaceous gland cell culture and treatment}

Supernatants of the immortalized human SZ95 sebocytes ${ }^{21}$ were used for the experiments. For lipid depletion of the supernatants Cleanascite lipid clarification reagent (Biotech Support Group, Monmouth Junction, NJ, USA) was used according to the manufacturer's instructions. Lipids; squalene, linoleic acid, oleic acid, palmitic acid and stearic acid (Sigma-Aldrich); were replaced individually subsequent to lipid depletion in a concentration of $150 \mu \mathrm{M}$. Replacement concentrations were determined by preliminary ELISA and flow cytometry measurements (Supplement Figure $1 \mathrm{a}$, Supplement Figure 3). Detailed protocol is available in supplementary information online.

\section{P. acnes strains}

Strain P. acnes 889 (P. acnes) was obtained from the Department of Microbiology, University of Debrecen, Hungary. Detailed protocol is available in supplementary information online.

This article is protected by copyright. All rights reserved. 


\section{Macrophage culturing and differentiation}

Monocytes were isolated from PBMCs using CD14 microbeads (Miltenyi Biotech, Vienna, Austria) following the manufacturer's protocol, cultured in RPMI 1640 media (Sigma-Aldrich) containing 10\% FBS (Biochrom, Berlin, Germany), 1\% antibiotics (Invitrogen, Carlsbad, CA, USA) and supplemented with SZ95 sebocyte supernatant. For further differentiation and activation IL-4, or $P$. acnes was used. Results presented are obtained with $80 \%$ SZ95 sebocyte supernatant supplementation after confirming dose dependence and an optimal dose as detailed in Supplement Figure 1b. Detailed protocol is available in supplementary information online.

\section{Immunohistochemistry}

For co-expression studies, the detection of Factor XIII subunit A (FXIII-A) was combined using antibodies against CD206, CD209 (Abcam, Cambridge, UK) and CD163 (Enzo Life Sciences, Farmingdale, NY, USA) antigens. Staining protocols and antibody specifications are detailed in supplementary information online (Supplement Table 1).

Flow cytometry

Macrophages were stained for surface markers CD206, CD209 using phycoerythrin (PE) conjugated mAbs (BD Bioscienses, New Jersey, NJ, USA). Data was collected by flow cytometric analysis using FACSCalibur (BD Bioscienses) and was analysed with Flowing Software (Cell Imaging Core, Turku, Finland). Staining protocols and antibody specifications are detailed in supplementary information online (Supplement Table 1).

This article is protected by copyright. All rights reserved. 


\section{Western blotting}

Macrophages were collected and processed for Western blot analysis. Proteins were probed with antiFXIII-A (Acris Antibodies, Herford, Germany) anti- $\beta$-actin (Cell Signaling, Danvers, MA, USA) and anti-IL-1B (R\&D Systems, Minneapolis, MN, USA). Detailed protocol as well as antibody specifications are available in supplementary information online (Supplement Table 1).

ELISA

IL-6, TNF-A and IL-1B cytokine levels were measured using the appropriate ELISA Duosets (R\&D Systems) according to the manufacturer's instructions. Detailed protocol is available in supplementary information online.

Phagocytosis assay

FITC-labelled $P$. acnes 889 treated macrophages were used for flow cytometric analysis using FACSCalibur (BD Bioscienses) and analysed with Flowing Software (Cell Imaging Core). Detailed protocol is available in supplementary information online.

\section{Statistics}

All data are presented as mean \pm SD. We made at least three biologic replicates for all experiments. Unpaired t-test, one-way ANOVA statistic test and Tukey post-hoc test were used in the analysis of ELISA data. Differences by $\mathrm{p}<0.05$ values were considered statistically significant.

This article is protected by copyright. All rights reserved. 


\section{Results}

Sebaceous gland rich skin is enriched in its dermal lipid content

As a starting point for our studies we first aimed to assess whether the lipid content of a given skin sample correlates with the presence of sebaceous glands. The used Oil-Red-O staining clearly demonstrated that in samples from sebaceous gland rich areas the lipid staining had an increased intensity in the dermis compared to samples from regions that were poor in sebaceous glands (Figure 1a). Interestingly, this increase was most prominent in the subepidermal part and around the sebaceous glands.

These results suggest that sebocytes might have a primary role in supplying the dermis with lipids.

\section{Sebum component lipids are able to penetrate through the epidermis and accumulate in the dermis}

Sebocytes secrete sebum to moisturize the skin surface. To assess the potential of sebum lipids to penetrate into the dermis we ex vivo selectively applied sebum component lipids onto human skin grafts from sebaceous gland poor regions of the body. By using Raman spectroscopy, we could show that each of the lipids accumulated in the dermis to varying degrees (Figure 1b).

These findings provide evidence that sebum lipids could have a far more complex physiological role than being only topical moisturizing agents, and call for further studies to investigate their effects on various cell types of the skin, such as macrophages.

SZ95 sebocytes promote alternative polarization of monocyte-derived macrophages

Activation and polarization of macrophages is greatly influenced by the stimuli from the tissue environment. Previously, we as well as others have shown that macrophages in healthy skin were typically expressing the markers of alternative activation (FXIII-A/CD209/CD206 $\left.{ }^{+}\right)^{22,23}$. Interestingly, we found that the macrophages accumulated in an increased number around the sebaceous glands ${ }^{24}$

This article is protected by copyright. All rights reserved. 
are exclusively alternatively activated in the healthy skin (Figure 2a), while in lesions of acne classically activated ones could also be detected (Supplement Figure 2). In order to test a possible interaction between sebocytes and macrophages, we established an in vitro model where peripheral blood monocytes were differentiated into macrophages in the presence of SZ95 sebocyte supernatant.

First, we characterized the effects of sebocytes on the polarization of macrophages and measured the expression of the widely-accepted markers via Western blotting (FXIII-A) and flow cytometry (CD206 and CD209). An enhancement of the expression of all markers studied was detected when monocytes were differentiated in the presence of the SZ95 sebocyte supernatant. Importantly, when using lipid depleted SZ95 sebocyte supernatant, we found a prominent down-regulation of FXIII-A and an almost complete inhibition in the induced expression of CD206 and CD209 pointing to a possible participation of sebaceous lipids in macrophage differentiation. Individually replacing the major components of the sebum such as oleic, linoleic, palmitic and stearic acids and squalene in the lipid-depleted SZ95 sebocyte supernatant used for macrophage culturing, we found linoleic and oleic acids to be potent inducers of the measured markers of alternative macrophage activation (Figure $2 b$ ), while squalene, stearic acid and palmitic acid had no effect (Supplement Figure 3).

Interestingly, we found that the high levels of CD206 and CD209 in response to IL-4 treatment, the primary stimulus to induce alternative activation, could be further increased when macrophages were cultured in SZ95 sebocyte supernatant (Supplement Figure 4). While, despite the strong polarizing effect towards the alternative activation, the SZ95 sebocyte supernatant treated macrophages conserved their potential to differentiate into classical activation suggesting that the polarizing effect can be overwritten in case of a danger signal. The co-treatment could neither relieve the down regulation of the measured markers nor the increased production of TNF-A in macrophages treated with IFN-G, the underlying stimulus behind the classical activation pathway (Supplement Figure 5).

This article is protected by copyright. All rights reserved. 
Sebocyte-derived lipids contribute to the potential of macrophages to uptake P. acnes

Alternatively activated macrophages have an enhanced potential for phagocytosis as one of their most important functional features. As $P$. acnes has been shown to contribute to the pathogenesis of acne, a disease marked by the inflammation of the pilosebaceous unit, we next measured the uptake of FITClabelled P. acnes by macrophages cultured with and without the SZ95 sebocyte supernatant. We found that in the presence of sebocyte-produced lipids macrophages gained an increased potential to uptake the bacteria. To address the possible lipids that may contribute to the enhanced phagocytosis, we selectively supplemented single lipids in the lipid-depleted SZ95 sebocyte supernatant and found linoleic and oleic acids to be potent players in the increased uptake (Figure 2c).

\section{Sebum component lipids contribute to the macrophage activating potential of $P$. acnes}

$P$. acnes is crucial in the pathogenesis of acne by inducing IL-1B production in macrophages, however, in in vitro settings, $P$. acnes itself only induces IL-1B production and secretion at a very moderate level suggesting that other factors are also required for its pathological role ${ }^{25,26}$. Therefore, we first measured if supplementation with the SZ95 sebocyte supernatant could alter the IL-1B levels in in vitro P. acnes-treated macrophages. Supporting an important role for sebocyte-derived products, a prominent induction in the protein levels of IL-1B was found when $P$. acnes was added in combination with SZ95 sebocyte supernatant to the culturing medium, as revealed by Western blot from cell lysates (Figure 3a). Interestingly, IL-1B production was further increased in the P. acnestreated macrophages when lipids were depleted from the SZ95 sebocyte supernatant (Figure 3 a).

To address the contribution of the previously tested lipids to the IL-1B secretion in $P$. acnes activated macrophages, of which palmitic acid is known to induce IL-1B via Toll-like receptor (TLR) 2, while

saturated fatty acids are recognised as TLR4 agonists ${ }^{27,28}$, we extended our studies by selectively replacing the different lipids in the lipid-depleted SZ95 sebocyte supernatant. Our ELISA measurements revealed that the lipids had different effects on the IL-1B secretion. Besides confirming the potential of palmitic acid, stearic acid, oleic acid and squalene to induce IL-1B, we could also This article is protected by copyright. All rights reserved. 
show that palmitic, oleic and stearic acids sensitized and further augmented the macrophage response to $P$. acnes. On the contrary, linoleic acid inhibited IL-1B production even in the presence of $P$. acnes (Figure $3 b$ ).

IL-6 and TNF-A are other characteristic inflammatory cytokines produced by infected macrophages. Measurement of these cytokines in the supernatants showed that the pro-inflammatory effect of stearic and palmitic acids and the anti-inflammatory effect of linoleic acid are not limited to IL1B secretion, but could also affect TNF-A secretion in a similar manner, while IL-6 was only slightly affected. Interestingly, oleic acid decreased the levels of both IL-6 and TNF-A, suggesting that it may have a special role among sebum lipids by selectively and differentially interacting with the different inflammatory signalling pathways (Figure $3 \mathrm{~b}, \mathrm{c}$ ).

These data show that the role of sebocytes in regulating macrophage function is not limited to altering their polarization, but can also be integrated into disease-specific settings, such as acne, where sebocytes may regulate inflammation via the secretion of its biologically active lipids (Figure 4).

\section{Discussion}

The concept of immunologically competent skin cells with no myeloid or lymphoid origin has been a challenging issue in skin biology. After decades of research keratinocytes, primarily via their cytokine production, are now accepted as immune regulators ${ }^{29-31}$ that are important in the pathogenesis of various inflammatory diseases, such as psoriasis and atopic dermatitis ${ }^{32,33}$. In contrast, sebaceous glands are still widely considered to contribute only to the lipid barrier of the skin by producing the

lipid rich sebum ${ }^{34,35}$, however, as we show in this work, there is an impressive difference already in the dermal lipid content between sebaceous gland rich and poor skin.

The key question put forward nearly 70 years ago, whether sebum lipids could contribute also to the dermal lipid content ${ }^{36}$, was first assessed by Butcher in the 1950s, who elegantly demonstrated that the sebum component lipids, stearic- (radioactive) oleic- and linoleic acids (with an increased

This article is protected by copyright. All rights reserved. 
fluorescence intensity) absorbed readily through rat skin with a notable accumulation around the sebaceous gland in the case of stearic acid, while linoleic acid passaged even to the blood vessels ${ }^{37,38}$. In this study, we extended and completed the characterization on the penetration of sebum component lipids by using Raman spectroscopy and showed that all the lipids (stearic-, linoleic-, oleic-, palmiticacids and squalene) were able to make their way through the epidermis. While Butcher proposed that lipids are mostly penetrating through the pilosebaceous duct, our results support that secreted sebum lipids from the skin surface could also penetrate and contribute to the dermal lipid milieu.

As lipids, besides cytokines and pathogens, also have a key role in the differentiation and activation of various immune cells, therefore, in our studies we aimed to challenge the interaction of sebocytes with macrophages, the key immune cells in maintaining the immune milieu of the healthy skin and also in initiating various diseases. Famous for their "Janus face", macrophages exert different functions depending on the tissue environment ${ }^{15}$. One extreme is their activation towards the alternative pathway, in which primarily IL-4/IL-13, but also different lipids might act as inducers ${ }^{14-}$ 16,39. This pathway, best represented by "skin resident macrophages" which express all the characteristic markers such as CD206, CD209 or FXIII-A, is central in the maintenance of the tissue environment by producing extracellular matrix components and contributing to tissue remodelling. On the other end of the spectrum is the classical activation, whose prototype is the formation of tuberculoid granulomas, where IFN-G and TNF-A are the key cytokines ${ }^{15}$. Integrating our findings into the current knowledge regarding macrophage activation pathways, we suggest that under normal conditions the exclusive presence of alternatively activated macrophages may be a sign and consequence of a continuous "non-danger signal" contributing to the homeostasis of the skin, of which sebocytes are a potential source ${ }^{3,12}$. However, based only on histological findings, it would be an over-interpretation to conclude a possible interaction between sebocytes and macrophages. Hence, we performed experiments with SZ95 sebocytes, which represent the best characterized model for human sebaceous gland research, including comparative and verified data on both lipid and protein synthesis $^{21,40}$, and raised the question whether sebocytes could interact with the maturation and polarization of macrophages. Considering the fact that sebocytes undergo a holocrine secretion, we

This article is protected by copyright. All rights reserved. 
only used cell free supernatant of SZ95 sebocytes to supplement the culture media of the human blood-derived monocytes differentiated into macrophages and showed that all the widely-accepted markers for the alternative activation pathway (CD206, CD209 and FXIII-A) were up-regulated. This suggests that sebocytes can, indeed, affect the polarization of macrophages favouring the alternative pathway and may also provide an answer for the phenomenon of macrophages expressing these detected markers in conditions where IL-4/IL-13 is not involved ${ }^{41}$. In addition, the supernatant-treated differentiated macrophages in vitro also exerted an increased capacity for phagocytosis of $P$. acnes, a previously defined hallmark of $\mathrm{CD} 209^{+}$macrophages ${ }^{42}$, suggesting that sebocytes might affect not just the marker profile but also functions of macrophages. These results support that sebocyte derived lipids could be potent contributors to the homeostasis of the skin which is in line with recent findings, that sebaceous gland rich healthy skin has a distinct non-inflammatory immune surveillance ${ }^{42}$.

Based on the findings, that the lipid composition of sebum is not a steady-state condition but can be changed, as it was observed both in in vitro sebocytes in response to various stimuli as well as in the sebum of acne patients where the variations in the quantity and mostly the quality of sebum lipids could significantly affect inflammation by targeting both keratinocytes and sebocytes ${ }^{4,43,44}$, we extended our experiments also with $P$. acnes-treated macrophages. Our results provided convincing data that macrophages could also be possible targets for the altered sebum production in acne. With dissecting the role of sebocyte-produced lipids in regulating the secretion of IL-1B and other inflammatory mediators, such as IL-6 and TNF-A by $P$. acnes-infected macrophages ${ }^{25,26,45}$, we found that each of the major lipid components had a well-defined inflammatory property, that has been overlooked so far perhaps not just in acne, but most likely in the pathogenesis of other dermatological diseases as well. Interestingly, palmitic and stearic acids, which are both major sebum components, were proven to be potent stimulators of inflammatory cytokine production in macrophages themselves even without the presence of $P . a^{a c n e s}{ }^{27}$. Moreover, our results that certain lipids, such as linoleic and oleic acids, had a significant effect on sensitizing macrophage response towards $P$. acnes even when the bacteria only had a minor effect on the secretion of IL-1B itself, addresses and (partially) explains another intriguing question, namely, how an otherwise commensal bacterium $P$. acnes, could still be a

This article is protected by copyright. All rights reserved. 
potent macrophage activator in diseases such as acne. Our results shed light on the essential role of the sebum lipids in this process. With ratio alterations, lipid fractions could determine the extent of macrophage' response to $P$. acnes and most likely to other pathogens as well. Keeping the limits of our study in mind, we also provide data supporting the biological relevance for the significant decrease in the levels of linoleic acid and thus the increased ratio of oleic/linoleic acid in the sebum of acne patients ${ }^{46}$ and suggest that linoleic acid may be a key player, not just in comedo formation, but also in the impaired symbiosis between pathogens and macrophages with a primarily antiinflammatory effect, which is further affected by the changing levels of the potent and selective IL-1B inducer oleic acid. These results altogether fully corroborate that sebaceous lipogenesis does not only contribute to the accumulation of sebum but is also an instrument for several coordinated actions ${ }^{12,47-}$ ${ }^{49}$, including the communication with macrophages and most likely with other immune cells that are yet to be investigated.

In conclusion, our results suggest that sebocytes, similarly to keratinocytes, may contribute to the inflammatory milieu and act as a guard at a "locus minoris resistentiae" within the dermis, especially for the pilosebaceous unit, where the lipids, cytokines and resident bacteria (that are all at elevated levels and unique to the pilosebaceous unit) form a unique symbiotic environment that has to be conserved and balanced. Moreover, based on our work, the analysis of sebum lipid fractions should also be addressed from the scope of their potential immunoregulatory functions, that may give new explanations for the altered lipid profile in sebum of various pathological conditions such as acne and rosacea $^{50,12,51}$. These findings altogether open several new avenues for research to consider lipids similarly as we focus on cytokines both in basic and therapeutic research.

\section{Conflict of interest}

The authors state no conflict of interest.

This article is protected by copyright. All rights reserved. 


\section{Acknowledgements}

Technical support in culturing the $P$. acnes 889 was kindly provided by Judit Szabó MD, PhD, Department of Medical Microbiology, University of Debrecen. We thank Tünde Toka-Farkas for technical assistance in PBMC isolation, Department of Dermatology, Faculty of Medicine, University of Debrecen and Sándorné Bana for technical support in fluorescent staining, Faculty of Public Health, University of Debrecen.

\section{References}

1 Strauss JS, Pochi PE, Downing DT. The sebaceous glands: twenty-five years of progress. $J$ Invest Dermatol 1976; 67: 90-7.

2 Thody AJ, Shuster S. Control and function of sebaceous glands. Physiol Rev 1989; 69: 383416.

3 Zouboulis CC, Baron JM, Bohm M et al. Frontiers in sebaceous gland biology and pathology. Exp Dermatol 2008; 17: 542-51.

4 Ottaviani M, Camera E, Picardo M. Lipid mediators in acne. Mediators Inflamm 2010; 2010.

5 Dobrosi N, Toth BI, Nagy G et al. Endocannabinoids enhance lipid synthesis and apoptosis of human sebocytes via cannabinoid receptor-2-mediated signaling. Faseb j 2008; 22: 3685-95.

6 Geczy T, Olah A, Toth BI et al. Protein kinase C isoforms have differential roles in the regulation of human sebocyte biology. J Invest Dermatol 2012; 132: 1988-97.

7 Toth BI, Geczy T, Griger Z et al. Transient receptor potential vanilloid-1 signaling as a regulator of human sebocyte biology. J Invest Dermatol 2009; 129: 329-39.

8 Torocsik D, Kovacs D, Camera E et al. Leptin promotes a pro-inflammatory lipid profile and induces inflammatory pathways in human SZ95 sebocytes. Br J Dermatol 2014.

9 Chen W, Tsai SJ, Wang CA et al. Human sebocytes express prostaglandin E2 receptors EP2 and EP4 but treatment with prostaglandin E2 does not affect testosterone production. $\mathrm{BrJ}$ Dermatol 2009; 161: 674-7.

10 Zhang Q, Seltmann H, Zouboulis CC et al. Activation of platelet-activating factor receptor in SZ95 sebocytes results in inflammatory cytokine and prostaglandin E2 production. Exp

Dermatol 2006; 15: 769-74.

This article is protected by copyright. All rights reserved. 
11 Zouboulis CC, Angres S, Seltmann H. Regulation of stearoyl-coenzyme A desaturase and fatty acid delta- 6 desaturase- 2 expression by linoleic acid and arachidonic acid in human sebocytes leads to enhancement of proinflammatory activity but does not affect lipogenesis. Br J Dermatol 2011; 165: 269-76.

12 Zouboulis CC, Jourdan E, Picardo M. Acne is an inflammatory disease and alterations of sebum composition initiate acne lesions. J Eur Acad Dermatol Venereol 2014; 28: 527-32.

13 Zouboulis CC, Picardo M, Ju Q et al. Beyond acne: Current aspects of sebaceous gland biology and function. Rev Endocr Metab Disord 2016; 17: 319-34.

14 Okabe Y, Medzhitov R. Tissue-specific signals control reversible program of localization and functional polarization of macrophages. Cell 2014; 157: 832-44.

15 Gordon S, Taylor PR. Monocyte and macrophage heterogeneity. Nat Rev Immunol 2005; 5: 953-64.

16 Sica A, Mantovani A. Macrophage plasticity and polarization: in vivo veritas. J Clin Invest 2012; 122: 787-95.

Rodrigues HG, Vinolo MA, Magdalon J et al. Oral administration of oleic or linoleic acid accelerates the inflammatory phase of wound healing. J Invest Dermatol 2012; 132: 208-15.

18 Alexeyev OA, Zouboulis CC. Shooting at skin Propionibacterium acnes: to be or not to be on target. J Invest Dermatol 2013; 133: 2292-4.

19 Szabo K, Erdei L, Bolla BS et al. Factors shaping the composition of the cutaneous microbiota. Br J Dermatol 2016.

20 Zouboulis CC. Propionibacterium acnes and sebaceous lipogenesis: a love-hate relationship? J Invest Dermatol 2009; 129: 2093-6.

21 Zouboulis CC, Seltmann H, Neitzel H et al. Establishment and characterization of an immortalized human sebaceous gland cell line (SZ95). J Invest Dermatol 1999; 113: 1011-20.

22 Torocsik D, Bardos H, Hatalyak Z et al. Detection of factor XIII-A is a valuable tool for distinguishing dendritic cells and tissue macrophages in granuloma annulare and necrobiosis lipoidica. J Eur Acad Dermatol Venereol 2014; 28: 1087-96.

Zaba LC, Fuentes-Duculan J, Steinman RM et al. Normal human dermis contains distinct populations of CD11c+BDCA-1+ dendritic cells and CD163+FXIIIA+ macrophages. J Clin Invest 2007; 117: 2517-25.

24 Christoph T, Muller-Rover S, Audring $\mathrm{H}$ et al. The human hair follicle immune system: cellular composition and immune privilege. Br J Dermatol 2000; 142: 862-73. NLRP3 inflammasome in human monocytes. J Invest Dermatol 2014; 134: 381-8.

26 Kistowska M, Gehrke S, Jankovic D et al. IL-1beta drives inflammatory responses to propionibacterium acnes in vitro and in vivo. J Invest Dermatol 2014; 134: 677-85.

This article is protected by copyright. All rights reserved. 
27 Snodgrass RG, Huang S, Choi IW et al. Inflammasome-mediated secretion of IL-1beta in human monocytes through TLR2 activation; modulation by dietary fatty acids. J Immunol 2013; 191: 4337-47.

28 Rocha DM, Caldas AP, Oliveira LL et al. Saturated fatty acids trigger TLR4-mediated inflammatory response. Atherosclerosis 2016; 244: 211-5.

29 Ansel J, Perry P, Brown J et al. Cytokine modulation of keratinocyte cytokines. J Invest Dermatol 1990; 94: 101s-7s.

30 Kupper TS. The activated keratinocyte: a model for inducible cytokine production by nonbone marrow-derived cells in cutaneous inflammatory and immune responses. $J$ Invest Dermatol 1990; 94: 146s-50s.

31 McKenzie RC, Sauder DN. The role of keratinocyte cytokines in inflammation and immunity. J Invest Dermatol 1990; 95: 105s-7s.

32 Giustizieri ML, Mascia F, Frezzolini A et al. Keratinocytes from patients with atopic dermatitis and psoriasis show a distinct chemokine production profile in response to T cellderived cytokines. J Allergy Clin Immunol 2001; 107: 871-7.

33 Lande R, Gregorio J, Facchinetti V et al. Plasmacytoid dendritic cells sense self-DNA coupled with antimicrobial peptide. Nature 2007; 449: 564-9.

34 Zouboulis CC. Is acne vulgaris a genuine inflammatory disease? Dermatology 2001; 203: 277-9.

35 Zouboulis CC. Sebaceous gland in human skin--the fantastic future of a skin appendage. $J$ Invest Dermatol 2003; 120: xiv-xv.

36 Calvery HO, Draize JH, Laug EP. The metabolism and permeability of normal skin. Physiol Rev 1946; 26: 495-540.

37 Butcher EO. The penetration of fat and fatty acid into the skin of the rat. J Invest Dermatol 1953; $21: 43-8$.

38 Butcher EO. Penetration of radioactive stearic acid into the skin of the rat. J Invest Dermatol 1953; 21: 243-7.

39 Torocsik D, Szeles L, Paragh G, Jr. et al. Factor XIII-A is involved in the regulation of gene expression in alternatively activated human macrophages. Thromb Haemost 2010; 104: 70917.

40 Dahlhoff M, Frohlich T, Arnold GJ et al. LC-MS/MS analysis reveals a broad functional spectrum of proteins in the secretome of sebocytes. Exp Dermatol 2016; 25: 66-7.

41 Fuentes-Duculan J, Suarez-Farinas M, Zaba LC et al. A subpopulation of CD163-positive macrophages is classically activated in psoriasis. J Invest Dermatol 2010; 130: 2412-22.

42 Liu PT, Phan J, Tang D et al. CD209(+) macrophages mediate host defense against Propionibacterium acnes. J Immunol 2008; 180: 4919-23.

This article is protected by copyright. All rights reserved. 
43 Downing DT, Stewart ME, Wertz PW et al. Essential fatty acids and acne. J Am Acad Dermatol 1986; 14: 221-5.

44 Dajnoki Z, Beke G, Kapitany A et al. Sebaceous Gland-Rich Skin Is Characterized by TSLP Expression and Distinct Immune Surveillance Which Is Disturbed in Rosacea. J Invest Dermatol 2017; 137: 1114-25.

45 Nagy I, Pivarcsi A, Kis K et al. Propionibacterium acnes and lipopolysaccharide induce the expression of antimicrobial peptides and proinflammatory cytokines/chemokines in human sebocytes. Microbes Infect 2006; 8: 2195-205.

46 Picardo M, Ottaviani M, Camera E et al. Sebaceous gland lipids. Dermatoendocrinol 2009; 1: 68-71.

47 Zouboulis. The sebaceous gland and its role as an endocrine organ., Vol. 1(1):37-51): JP Medical Publishers. 2013.

48 Lee SE, Kim JM, Jeong MK et al. 11beta-hydroxysteroid dehydrogenase type 1 is expressed in human sebaceous glands and regulates glucocorticoid-induced lipid synthesis and toll-like receptor 2 expression in SZ95 sebocytes. Br J Dermatol 2013; 168: 47-55.

49 Makrantonaki E, Zouboulis CC. Testosterone metabolism to 5alpha-dihydrotestosterone and synthesis of sebaceous lipids is regulated by the peroxisome proliferator-activated receptor ligand linoleic acid in human sebocytes. Br J Dermatol 2007; 156: 428-32.

50 Camera E, Ludovici M, Galante $\mathrm{M}$ et al. Comprehensive analysis of the major lipid classes in sebum by rapid resolution high-performance liquid chromatography and electrospray mass spectrometry. J Lipid Res 2010; 51: 3377-88.

51 Ni Raghallaigh S, Bender K, Lacey $\mathrm{N}$ et al. The fatty acid profile of the skin surface lipid layer in papulopustular rosacea. Br J Dermatol 2012; 166: 279-87.

\section{Figure legends}

Figure 1. Oil-Red-O staining of sebaceous gland poor (stomach; left) and sebaceous gland rich (nose; right) skin. Arrows indicate sebaceous glands, asterisks indicate lipid rich areas in the subepidermal region, while diamond arrows point at the epidermal lipid barrier. Note, that in the sebaceous gland (SG) rich sample an increased staining was observed not just in the vicinity of the SGs but also beneath the epidermis. The comparable intensities in the epidermal staining of the SG rich and poor samples supports that keratinocytes are important in the epithelial lipid barrier, showing no correlation with the dermal lipid content (a).

This article is protected by copyright. All rights reserved. 
Raman map of control versus squalene (SQ), stearic- (STA), palmitic- (PA), linoleic- (LA) or oleic acid $(\mathrm{OA})$ treated skin samples. From blue to red colouring the map indicates the increasing presence of the various fatty acids in a semi-quantitative manner. Dotted lines mark the estimated dermoepidermal border (b).

\section{Figure 2. SZ95 sebocytes promote alternative activation of monocyte-derived macrophages}

Double immunofluorescent labelling for FXIII-A (green) and CD206, CD209 (red) in healthy skin samples detecting macrophages around the sebaceous glands to be alternatively activated (orange) $\left(\mathrm{FXIII}^{+} \mathrm{A}^{+} / \mathrm{CD} 206^{+} / \mathrm{CD} 209^{+}\right.$). Scale bar $100 \mu \mathrm{m}$ and $50 \mu \mathrm{m}$ (a). Western blot analysis of FXIII-A protein levels $(n=3)$ and flow cytometric analysis of CD206, CD209 cell surface markers $(n=5)$ is depicted in in vitro differentiated macrophages in the presence SZ95 sebocyte supernatant containing lipids (SZ95 sup.), after lipid depletion (lipid depleted SZ95) or supplementation with various sebum lipids (squalene, linoleic-, oleic-, stearic- or palmitic acids). Supplementation of lipid-depleted SZ95 sebocyte supernatant with linoleic (LA) and oleic (OA) acids partially restored the induction of CD206 and CD209 (b). Contribution of sebocyte-derived lipids to the uptake of FITC-labelled $P$. acnes by macrophages was assessed when macrophages were cultured in the presence of SZ95 sebocyte supernatant, lipid-depleted SZ95 sebocyte supernatant or lipid-depleted SZ95 sebocyte supernatant supplemented with linoleic- (LA) or oleic (OA) acid (n=3) (c).

\section{Figure 3. SZ95 sebocyte supernatant modifies the macrophage activating potential of $P$. acnes}

IL-1B production in in vitro cultured $P$. acnes treated macrophages with and without SZ95 sebocyte supernatant. Note that $P$. acnes induced IL-1B production only in the presence of SZ95 sebocyte supernatant which could be further increased when lipids were depleted from the SZ95 sebocyte supernatant as revealed by Western blot $(n=3)$ Dosimetry data depicts the relative differences in IL1B production of macrophages as a result of P.acnes treatment differentiated in the presence of

This article is protected by copyright. All rights reserved. 
complete or lipid depleted (LD) SZ95 sebocyte supernatant (a). IL-1B secretion of macrophages differentiated in the presence of lipid depleted SZ95 sebocyte supernatant supplemented with $P$. acnes and/or the indicated lipids were analysed for IL-6, TNF-A and IL-1B from their supernatants by ELISA. Mean \pm SD of samples assayed in duplicate is depicted $(\mathrm{n}=3) ; * \mathrm{p} \leq 0.05, * * \mathrm{p} \leq 0.01, * * *$ $\mathrm{p} \leq 0.001$; SQ: Squalene, SA: Stearic acid, PA: Palmitic acid, LA: Linoleic acid, OA: Oleic acid (b). Heat map summarizing the IL-1B, IL-6 and TNF-A secretion of macrophages (with and without $P$. acnes stimuli) to display the selective inflammatory effects of the various sebum component lipids on differentiated and activated macrophages (c).

\section{Figure 4. Overview figure on the possible role of sebocytes in modulating macrophage activation, differentiation and function}

Sebocytes are able to contribute to the polarization of monocytes towards the alternative activation with their produced lipids, as marked by increased levels of CD206, CD209 and FXIII-A expression, of which linoleic and oleic acids are essential. Furthermore, linoleic and oleic acids contribute to an increased potential of macrophages to uptake P. acnes, while palmitic-, oleic- and stearic acids augment the macrophage response to the bacteria.

This article is protected by copyright. All rights reserved. 
Figure 1.
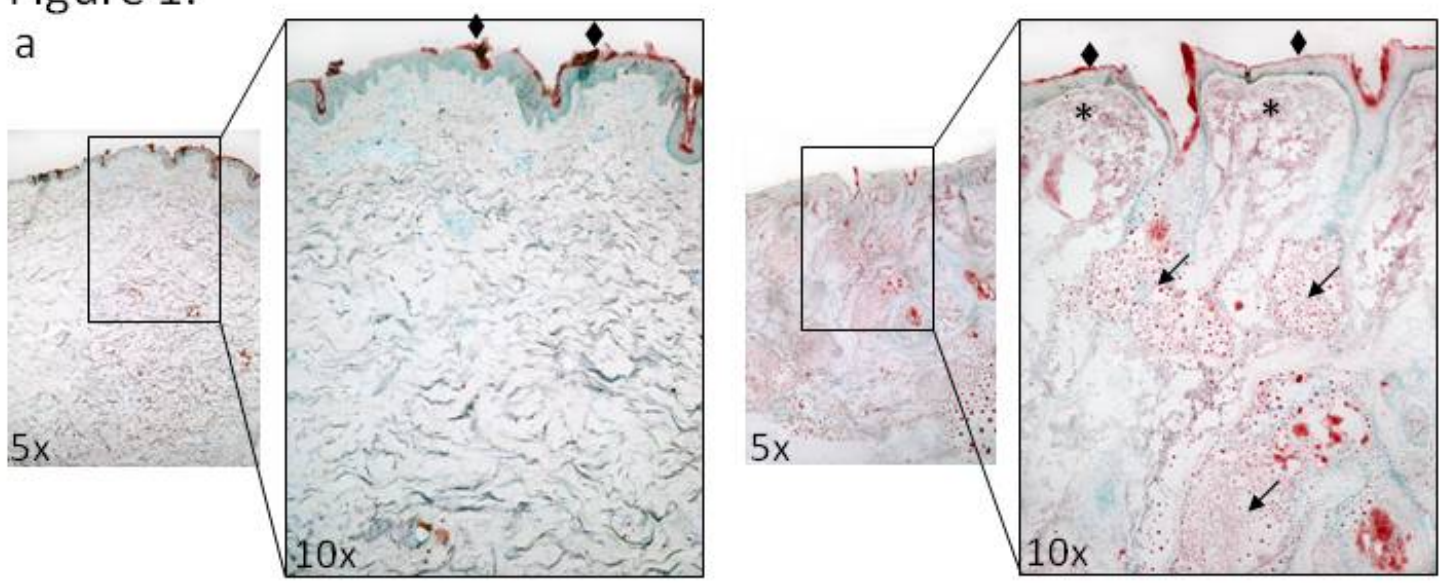

b
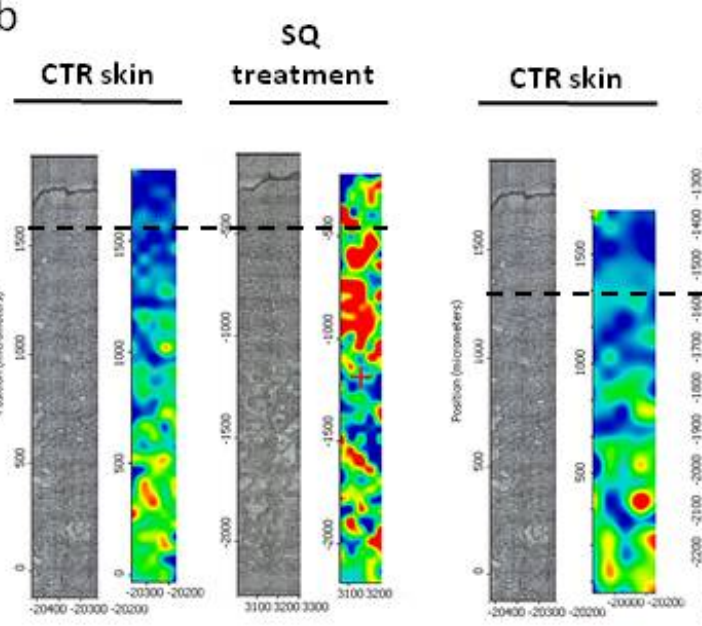

STA
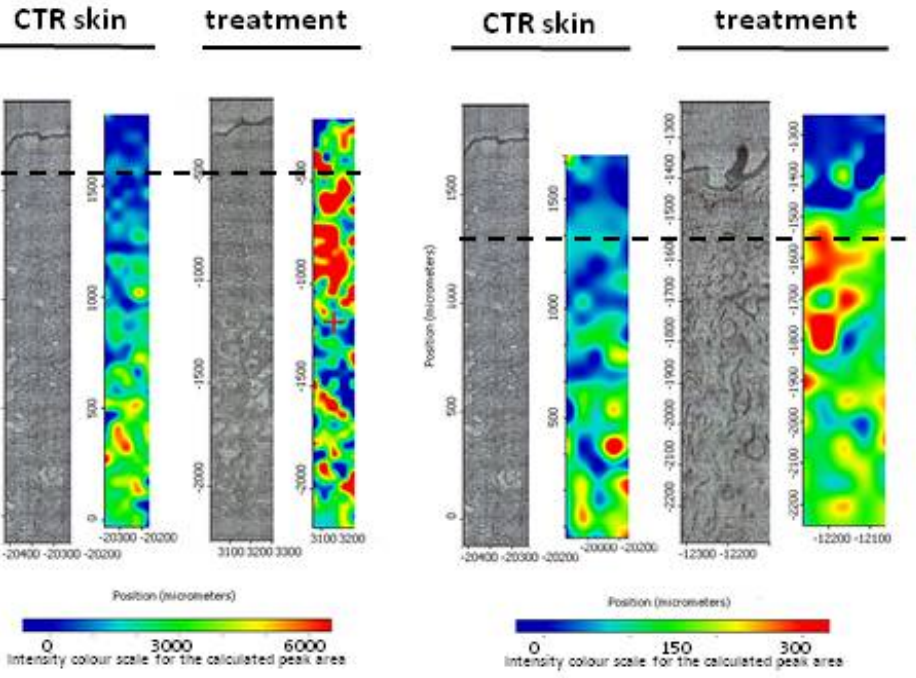

LA
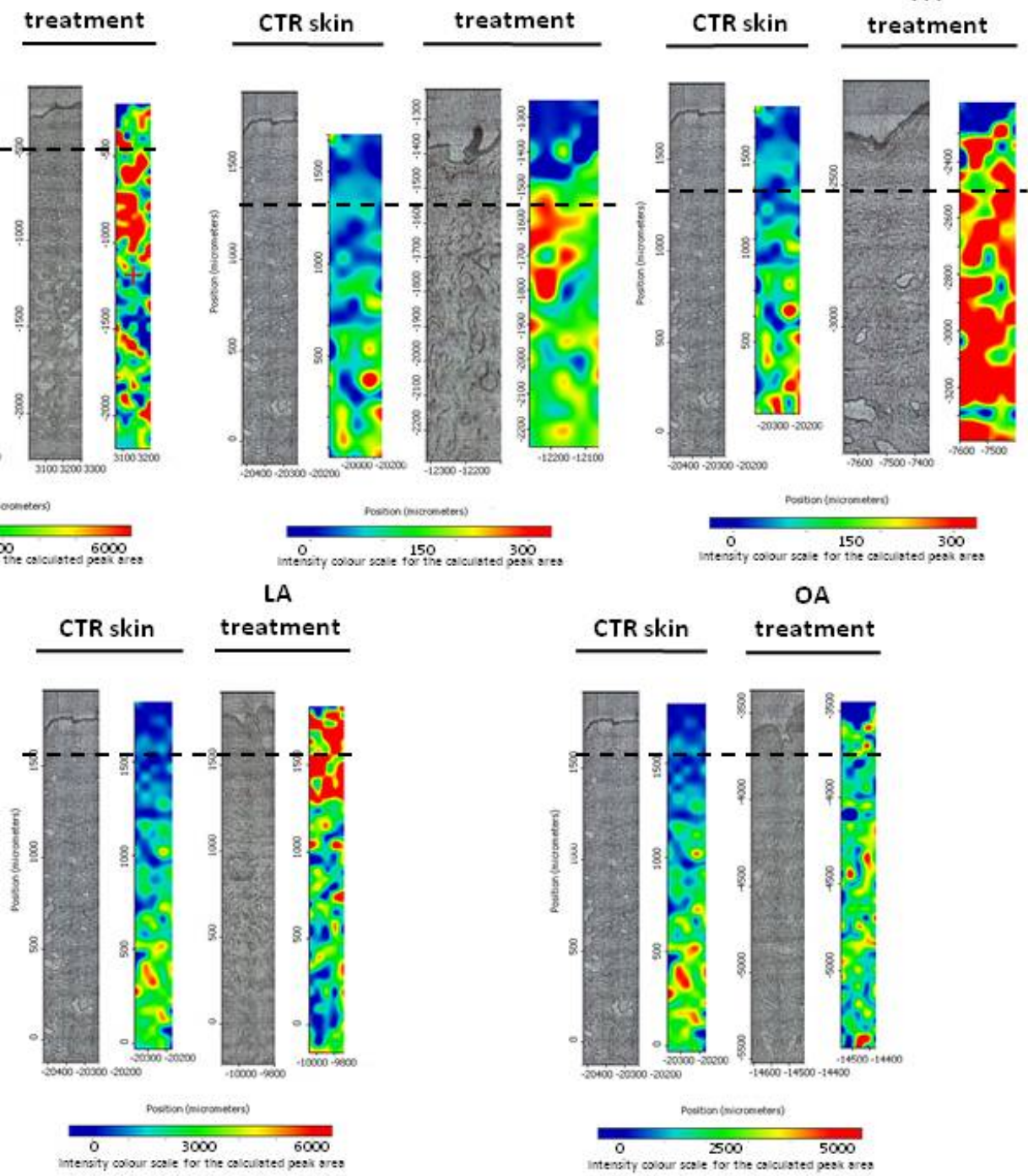

This article is protected by copyright. All rights reserved. 
Figure 2.

a.
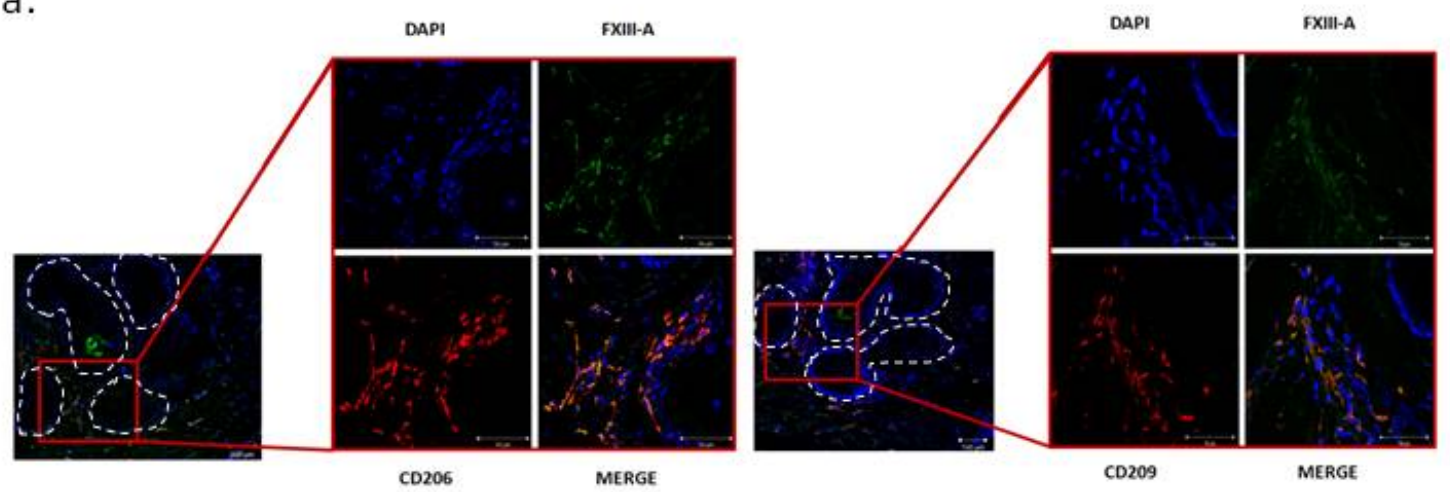

b.
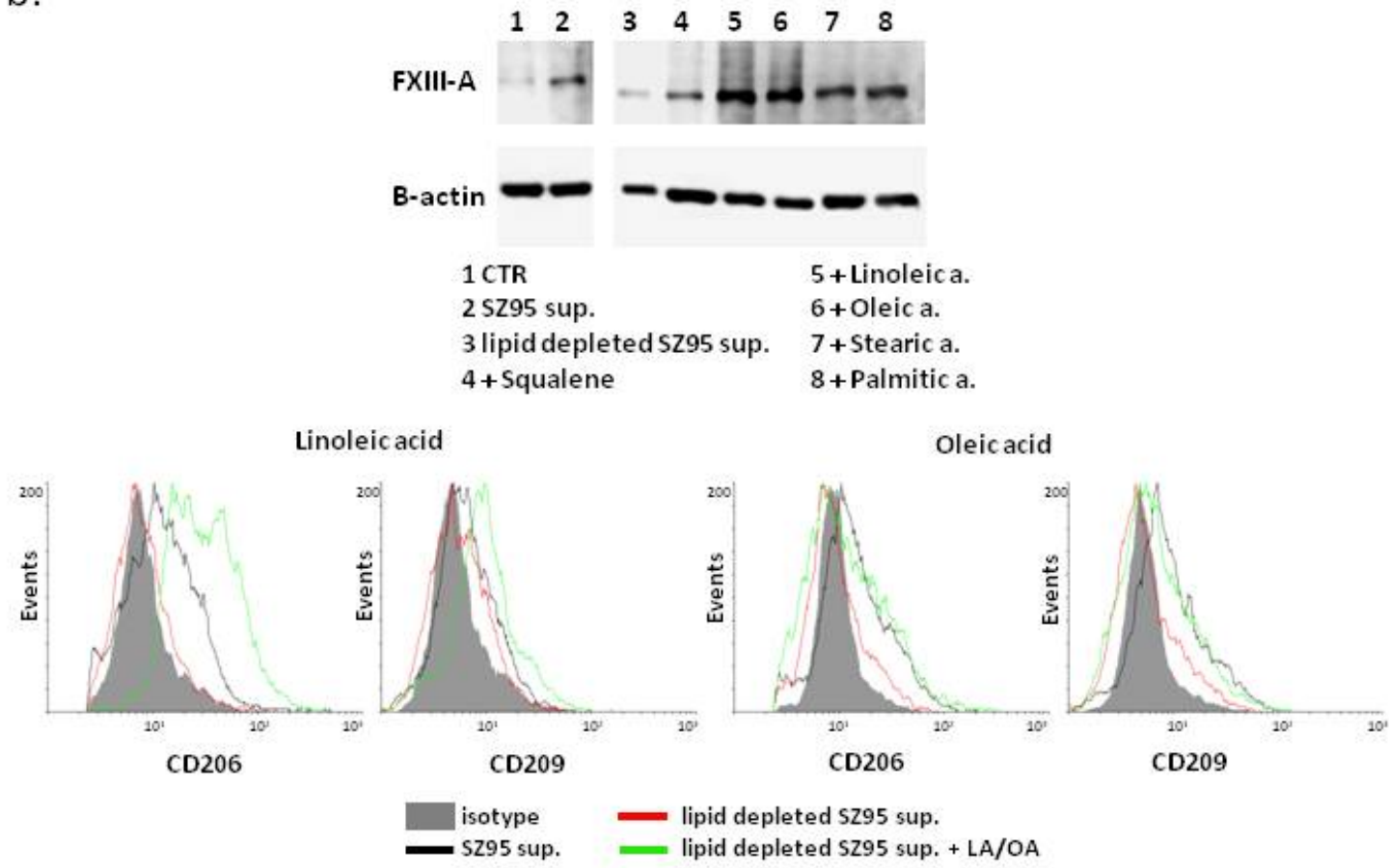

C.
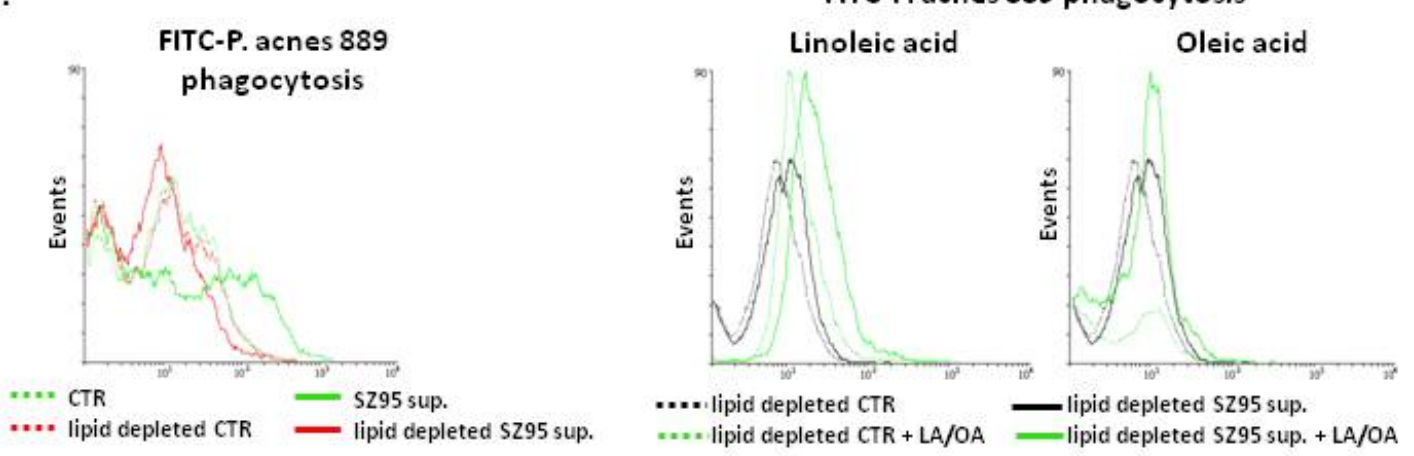

This article is protected by copyright. All rights reserved. 
Figure 3.
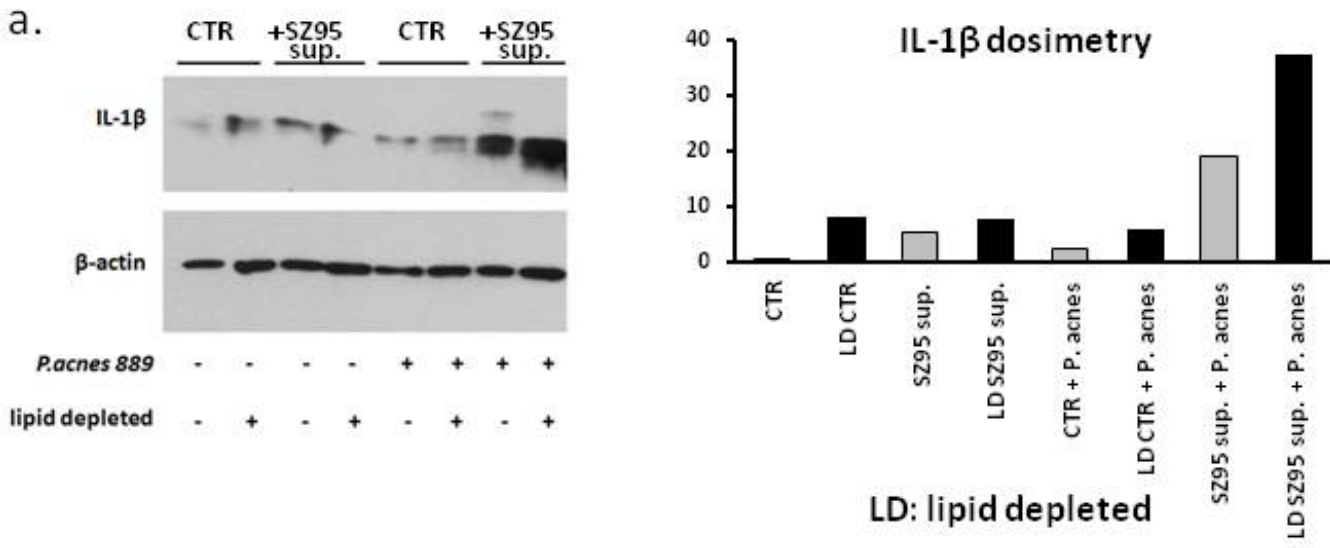

b.
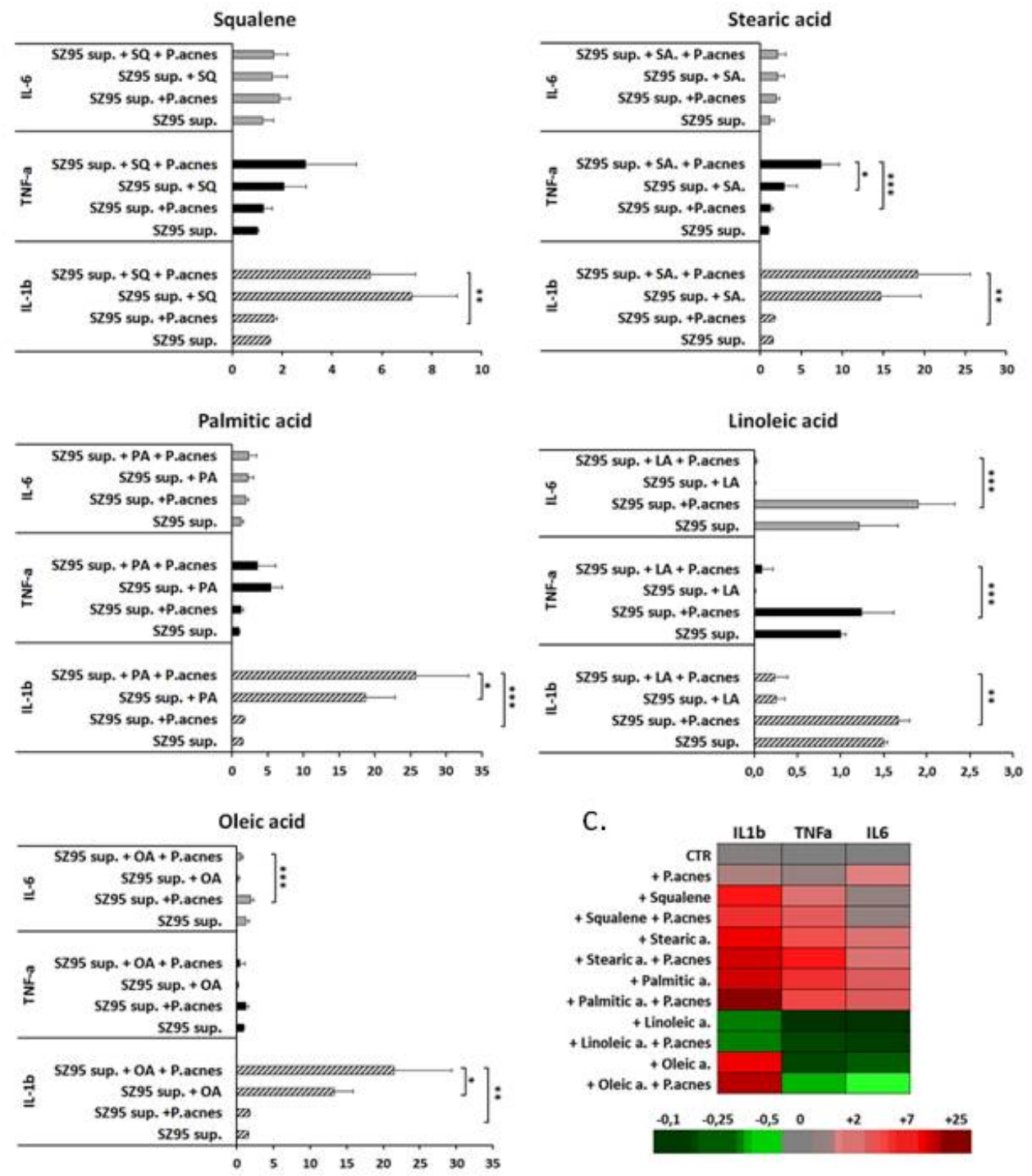

This article is protected by copyright. All rights reserved. 
Figure 4.

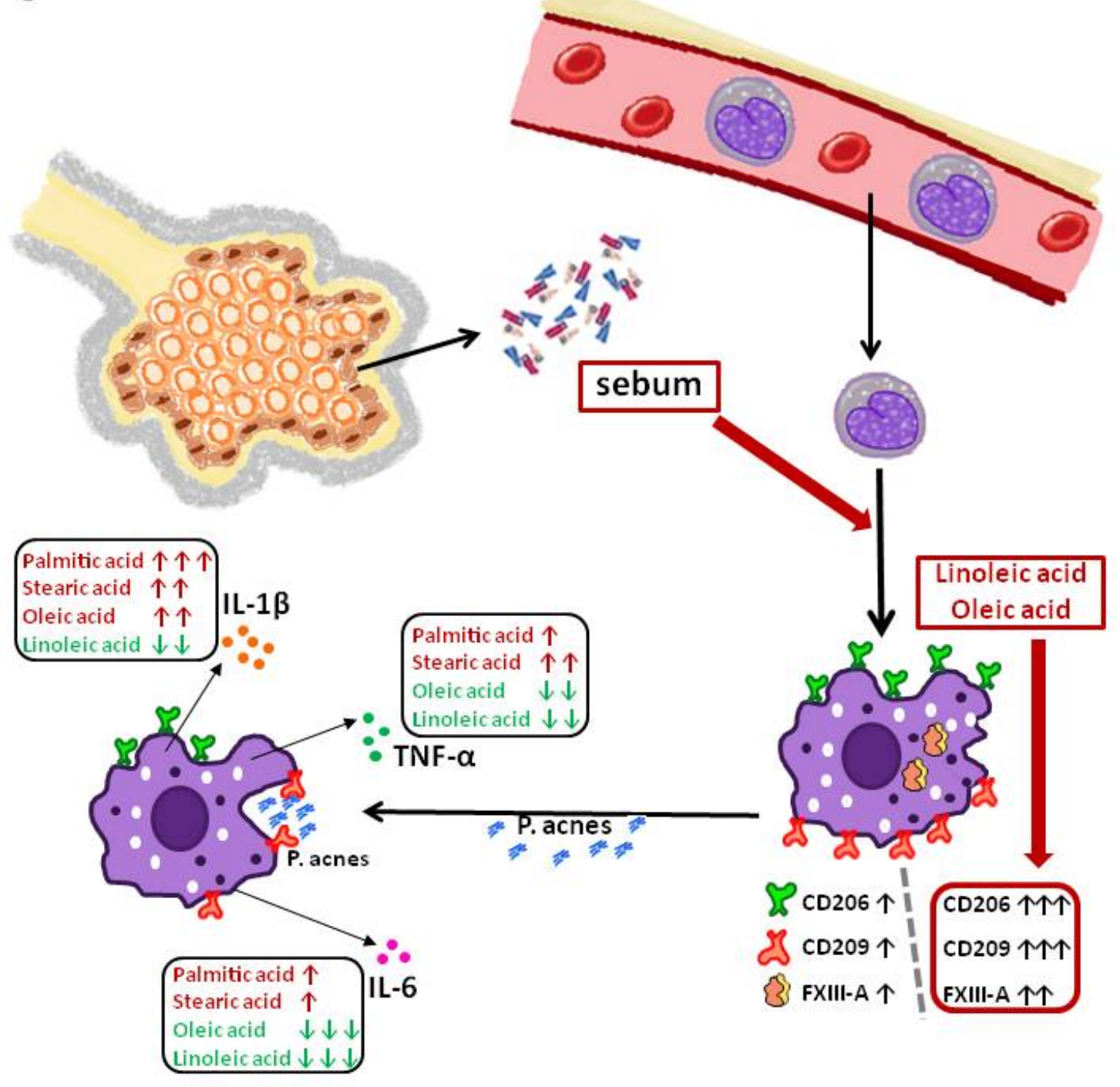

This article is protected by copyright. All rights reserved. 